\title{
Phase Coding of RF Pulses in Photonics-Aided Frequency-Agile Coherent Radar Systems
}

\author{
Paolo Ghelfi, Filippo Scotti, Francesco Laghezza, and Antonella Bogoni
}

\begin{abstract}
An innovative optical scheme to generate software-defined phase-modulated radio frequency (RF) pulses with carrier frequency agility from a mode-locked laser (MLL) is proposed. The technique exploits a direct digital synthesizer and a Mach-Zehnder modulator to apply an intermediate frequency modulation to the MLL's modes. The heterodyne detection of the optical signal allows the generation of amplitude- and phase-modulated RF carriers with very high phase stability, suitable for coherent radar applications. Further, a single MLL can be used to generate carriers simultaneously at different frequencies, enabling frequency hopping or multifunctional radars, with no need to increase the complexity of the transmitter. Results show chirped and Barker-coded pulses at around 10 or $40 \mathrm{GHz}$ in a single setup, without any performance degradation while increasing the carrier frequency. The proposed technique allows the practical realization of compressed pulses for coherent radars over a wide carrier frequency range, allowing the development of software-defined radar systems with improved functionalities.
\end{abstract}

Index Terms-Barker code, chirp, coherent radar, laser mode-locking, microwave photonics, phase modulation.

\section{INTRODUCTION}

$\mathbf{T}$ HE EVOLUTION of coherent radars is requiring, among other technical developments, the improvement of the system sensitivity and the reduction of the antennas dimensions. To these aims, RF carriers with lower phase noise and higher frequency are required. Moreover, radar systems are seeking increased resolution to detect and recognize small objects, and to this purpose, rather than reducing the duration of the radar pulses, pulse compression techniques have been implemented which broaden the signal bandwidth by modulating or coding the phase of the radar pulse carrier, thus avoiding the transmission of dangerous high-power RF pulses [1].

To increase the detection probability and to reduce the risk of false detections in adverse conditions, the transmission of two or more radar signals at different carrier frequency is often implemented, thus reducing the target signature fluctuations and averaging and smoothing the complex echo signals. This technique, called frequency agility (or diversity), also implies

Manuscript received December 19, 2011; revised June 7, 2012; accepted June 15, 2012. Date of publication June 20, 2012; date of current version July 2, 2012. This work was supported in part by the EU Projects PHODIR and ACEPLAN.

The authors are with the National Interuniversity Consortium for Telecommunications (CNIT), Pisa 56124, Italy (e-mail: paolo.ghelfi@cnit.it; filippo.scotti@cnit.i; francesco.laghezza@iet.unipi.it; antonella.bogoni@ cnit.it).

Color versions of one or more of the figures in this paper are available online at http://ieeexplore.ieee.org.

Digital Object Identifier 10.1109/JQE.2012.2205371 additional benefits. In fact, it allows the elimination of the Doppler blind speed and of the Doppler dilemma. In weather radars it can be used to build up a composite image of clouds and rainfalls. Frequency agility is also useful for jamming immunity, making more difficult to disturb the radar and, if the system rapidly switches between several frequencies, can render the jamming ineffective. Finally, radar systems with multiple carriers are becoming of interest in dual-band multifunctional radars, where surveillance and detection \& tracking are implemented simultaneously using a single radar apparatus [2].

The high phase stability required in modern radar systems is becoming challenging for the electronic RF signal generation, since at high carrier frequency the frequency multiplication processes that are usually exploited reduce the phase stability of the original RF oscillators. On the other hand, frequency diversity radars are commonly realized by using two or more radar transmitters, implying a significant increase in cost and power consumption [3].

In the last years the use of photonics has been suggested as an effective way for generating low phase-noise RF carriers even at high frequency. Among other techniques [4], [5], the heterodyning of modes from a mode locking laser (MLL) has proven to generate low-phase noise RF carriers up to the EHF band (30-300GHz) [6]. The photonics-generated carriers could then be modulated into phase-coded radar pulses via electronics methods, which would require frequency-specific RF components, e.g., the mixers, that become more expensive at increasing frequency. An alternative modulation and coding approach based on photonics techniques could instead allow an agile carrier frequency selection without RF bandwidth restrictions. Few example of this approach have been proposed so far, based on wavelength-to-time conversion [7]-[9], on microwave photonic filters [10], or on the heterodyning of phase modulated continuous-wave lasers [11]. These works are all based on rather environmental sensitive setups, as free space propagation or fiber interferometers, and therefore are not suitable for coherent radar applications. Recently we have proposed a more robust architecture that exploits an optical I/Q modulator in a peculiar configuration, demonstrating the generation of radar signals with different pulse compression techniques [12]. But this scheme suffers of limited modulation bandwidth. Moreover, none of the work cited above has been demonstrated to allow the frequency agility needed in coherent radar systems, although a similar concept was expressed in 2002 [13].

In this paper we propose an innovative technique for optically generating phase-coded RF pulses with flexible carrier 


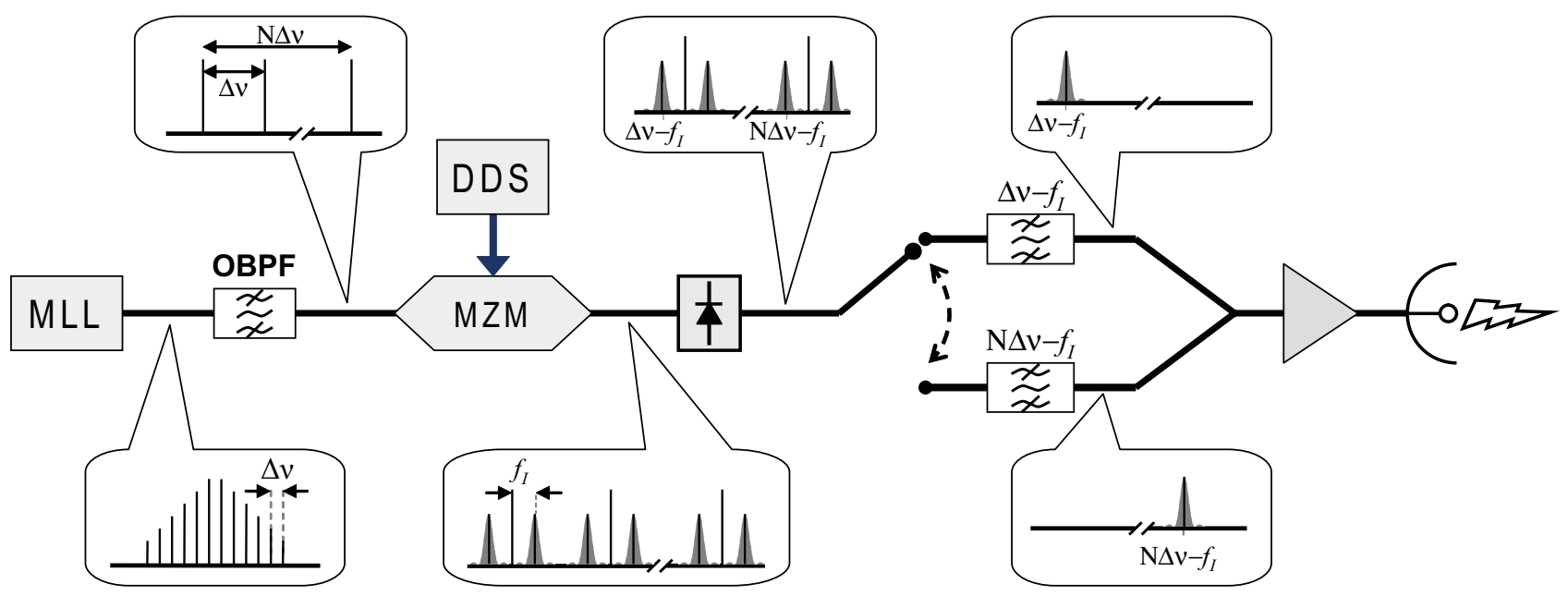

Fig. 1. Experimental setup. $\Delta v=10 \mathrm{GHz}, N=4$, and $f_{I F}=100 \mathrm{MHz}\left(\Delta v-f_{I F}=9.9 \mathrm{GHz}\right)$ or $f_{I F}=184 \mathrm{MHz}\left(N \Delta v-f_{I F}=39.816 \mathrm{GHz}\right)$.

frequency and, thanks to the use of a MLL, with a phase stability suitable for coherent radar systems. The idea is to optically modulate the modes of a MLL at intermediate frequency, so that RF signals at any multiple frequency of the MLL repetition rate can be obtained by heterodyning in a photodiode the modulated MLL modes. The use of a direct digital synthesizer (DDS) for generating the coding signals at intermediate frequency allows the implementation of a software-defined radar transmitter, matching the requirements of next detection and surveillance systems. Experiments are also reported, showing the realization of some of the most common pulse-compression techniques used in radar applications (i.e. frequency chirp and Barker-codes), applied to RF pulses at around $10 \mathrm{GHz}$ and $40 \mathrm{GHz}$ exploiting the same setup. The results confirm the high phase stability, and the analysis on transients and spectra prove the effectiveness of the applied modulations.

\section{PRINCIPLE OF OPERATION}

To optically generate a phase-modulated RF signal at a specific carrier frequency $f_{c}$, it is necessary to heterodyne two stable continuous-wave $(\mathrm{CW})$ lasers with a frequency detuning $\Delta v=f_{c}$, one of which must be phase modulated. The practical problem in this procedure is to process the two lasers independently without affecting their reciprocal phase stability. In fact, if both the lasers are modulated by a baseband signal in the same setup, the phase information would be lost after the heterodyning. In [12] we have solved the problem exploiting an optical I/Q modulator to simultaneously generate new CW components on one arm of the modulator, and modulate the phase of the original lasers on the other arm. Here we simplify that approach by directly generating new spectral components which are phase-modulated. In fact, if two lasers are modulated by a signal centered at intermediate frequency $f_{I F}$, new sidebands are generated in the optical spectrum at $\pm f_{I F}$ from each laser. If the original laser carriers are not suppressed during the modulation process, after the photodetection several components are present, including those centered at frequency $\Delta v \pm f_{I F}$ that are the beating between the original unmodulated laser modes and the newly generated sidebands, which bring the desired modulation including the phase. Therefore an RF filter centered at $\Delta v \pm_{I F}$ will permit to select the desired RF signal.

This scheme can be easily extended to generate flexible carrier radar signals if more than two CW lasers (e.g., the laser comb provided by a MLL) are modulated by the signal centered at $f_{I F}$ and heterodyned in a photodiode with sufficient bandwidth (see Fig. 1). In this case, phase-modulated RF signals are generated at $\pm f_{I F}$ from any beating frequency between the considered lasers. Then a set of RF filters can be used to select the signal at the desired carrier frequency. A similar approach was used in [12].

The operation of modulating the RF carriers with a signal at intermediate frequency must not affect the phase stability of the unmodulated carriers. An interesting solution is generating the modulating signal with a low-frequency high-quality direct digital synthesizer (DDS), which also allows to flexibly change the modulating waveform and the intermediate frequency, thus realizing a software-defined radar signal.

To better explain the principle of operation, in particular when the laser comb from a MLL is exploited, we add here a more formal description of the involved signals. If we take into account a series of $N+1$ laser modes produced by a MLL, the time domain signal can be expressed as

$$
S_{M L L}(t)=\sum_{n=0}^{N} A_{n} \cos [2 \pi(v+n \Delta v) t]
$$

where $\mathrm{A}_{n}$ is the modes amplitude, $v$ represent the mode optical frequency, and $\Delta v$ is the mode spacing given by the laser repetition rate.

Let's consider a modulating signal centered at intermediate frequency, whose time domain expression is:

$$
S_{I F}(t)=\operatorname{rect}_{T}(t) \cos \left[2 \pi f_{I F} t+\varphi(t)\right]
$$

where $f_{I F}$ is the intermediate frequency (IF), $\phi(t)$ is the phase modulation carried on the IF, and the function $\operatorname{rect}_{T}(t)$ represents the pulsed signal with a limited time duration $\mathrm{T}$ and bandwidth $B=1 / T$ (the typical shape of a radar pulse). 
The amplitude of the optical signal $S_{M L L}(t)$ is modulated by the signal $S_{I F}(t)$ (with a modulation depth $M$ ):

$$
S_{M O D}(t)=S_{M L L}(t) \cdot\left[1+M \cdot S_{I F}(t)\right]
$$

that becomes

$$
\begin{aligned}
S_{M O D}(t)=\sum_{n=0}^{N}\left\{B_{n} \cos [2 \pi(v+n \Delta v) t]\right. \\
+C_{n} \cos \left[2 \pi\left(v+n \Delta v \pm f_{I F}\right) t\right. \\
\left.+\varphi(t)] \cdot \operatorname{rect}_{T}(t)\right\}
\end{aligned}
$$

with $\mathrm{B}_{n}$ and $\mathrm{C}_{n}$ amplitude terms.

After the heterodyning in a photodiode, we obtain the beatings of all the different carriers, i.e.

$$
\begin{gathered}
S_{P D}(t)=\sum_{n=0}^{N}\left\{D_{n} \cos \left[2 \pi\left(n \Delta v \pm f_{I F}\right) t+\varphi(t)\right] \operatorname{rect}_{T}(t)\right. \\
\left.+E_{n} \cos (2 \pi n \Delta t)\right\}+[\ldots]
\end{gathered}
$$

where the first term represents the phase-modulated signal upconverted to $\mathrm{n} \Delta v \pm f_{I F}$, and the second term represents the beatings between the unmodulated carriers; $\mathrm{D}_{n}$ and $\mathrm{E}_{n}$ are the amplitudes of the components; [...] denotes the omitted beatings between all the sidebands at $v+n \Delta v \pm f_{I F}$, the component at DC, and the components at twice the optical frequency. A graphical representation of the optical components at the input of the photodiode and of the beatings of interest is reported in Fig. 2.

The frequency carrier desired for transmission can be finally selected by exploiting an RF filter with central frequency $\mathrm{n} \Delta v-f_{I F}\left(\right.$ or $\left.\mathrm{n} \Delta v+f_{I F}\right)$, with $0<\mathrm{n}<\mathrm{N}$ :

$$
S_{T X}(t)=\cos \left[2 \pi\left(n \Delta v-f_{I F}\right) t+\varphi(t)\right] \operatorname{rect}_{T}(t)
$$

\section{EXPERIMENTAL SETUP}

Fig. 1 reports the exploited experimental setup. The laser source is a regenerative fiber MLL with a repetition rate of $10 \mathrm{GHz}$, an optical bandwidth of $250 \mathrm{GHz}$, and an output power of about $13 \mathrm{dBm}$. An optical band-pass filter (OBPF) with a bandwidth of $0.4 \mathrm{~nm}$ selects a bunch of about five adjacent modes, which are launched into a Mach-Zehnder modulator (MZM) biased at its quadrature point. The optical modes are modulated by the electrical signal generated by a 16-bit 400Msample/s DDS. The modulating signal is a phase-coded RF pulse centered around $f_{I F}=100 \mathrm{MHz}$ or $f_{I F}=184 \mathrm{MHz}$, which will be used to generate RF signals respectively at $9.9 \mathrm{GHz}$ and $39.816 \mathrm{GHz}$, as explained in the following. The RF pulse has a duration of $5 \mu \mathrm{s}$ and a repetition time of $15 \mu \mathrm{s}$. In the experiment, two different phase codes will be used, the linear chirp and the Barker code, which will be described in the following. Fig. 3 reports a time domain representation (at an arbitrary low frequency, for the sake of clarity) of the RF pulse in the two considered case.

The modulation produces sidebands around each laser modes, and when the optical signal thus generated is detected by a $50 \mathrm{GHz}$-bandwidth photodiode, all the beatings between modes and sidebands are produced. Two RF filters with a bandwidth of $40 \mathrm{MHz}$ and centered at $9.9 \mathrm{GHz}$ and at

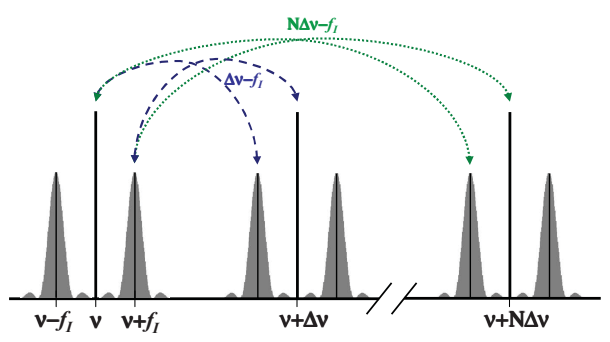

Fig. 2. Optical spectrum before the photodiode. The beatings of interest are highlighted.

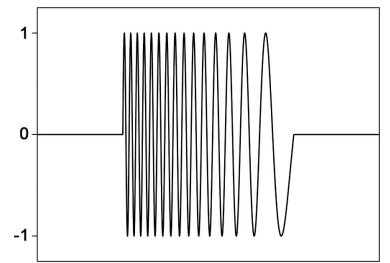

(a)

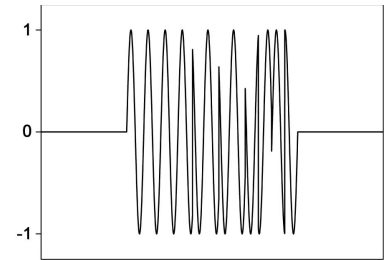

(b)
Fig. 3. Time domain representation (at an arbitrary low frequency) of the applied phase-coded RF pulses generated by the DDS, in the cases of (a) chirp code and (b) Barker code.

$39.816 \mathrm{GHz}$ are then used to alternately select the phasemodulated signal when $f_{I F}$ is respectively equal to $100 \mathrm{MHz}$ and $184 \mathrm{MHz}$ (in order to match the center frequency of the filters), and a broadband electrical amplifier with a gain of $27 \mathrm{~dB}$ allows to perform the analyses.

\section{EXPERIMENTAL RESULTS}

First, the phase noise of the signal at $9.9 \mathrm{GHz}$ is analyzed to study the effect of the modulation at $f_{I F}$ on the phase stability of the carrier. The frequency-shifted signal, generated with the DDS programmed to emit only a sinusoid at $100 \mathrm{MHz}$, is measured by means of a signal source analyzer (SSA), and compared with the signal generated by the beating of the original laser modes at $10 \mathrm{GHz}$, and with the phase noise of the shifting signal at $100 \mathrm{MHz}$. The phase noise curves are reported in Fig. 4(a). As shown by the graphs, the signal generated by the low-frequency high-quality DDS presents a lower phase noise than that of the carrier at 10GHz. Therefore the frequency shift induced on the optical modes does not affect the stability of the desired carrier at $9.9 \mathrm{GHz}$, which shows the same behavior as the non-shifted one. Only a higher noise floor is present for frequency offset above $100 \mathrm{kHz}$, which is due to the higher thermal noise in the measure and can be reduced with a more accurate amplification.

In order to compare the phase stability achievable with the proposed scheme to a conventional electronic generation, Fig. 4(top) also reports the phase noise of a signal at $9.9 \mathrm{GHz}$ generated by mixing the $100 \mathrm{MHz}$ signal from the DDS with a carrier at $10 \mathrm{GHz}$ from a state-of-the-art RF synthesizer (Agilent E8257D). The optically generated carrier shows a lower phase noise for offset frequency from $2 \mathrm{kHz}$ to $200 \mathrm{kHz}$, confirming the quality of the photonics-based approach. The higher noise below $2 \mathrm{kHz}$ is due to the mechanical fluctuations of the exploited fiber-based MLL, but it is possible to strongly reduce this noise if the MLL is locked to a reference clock 


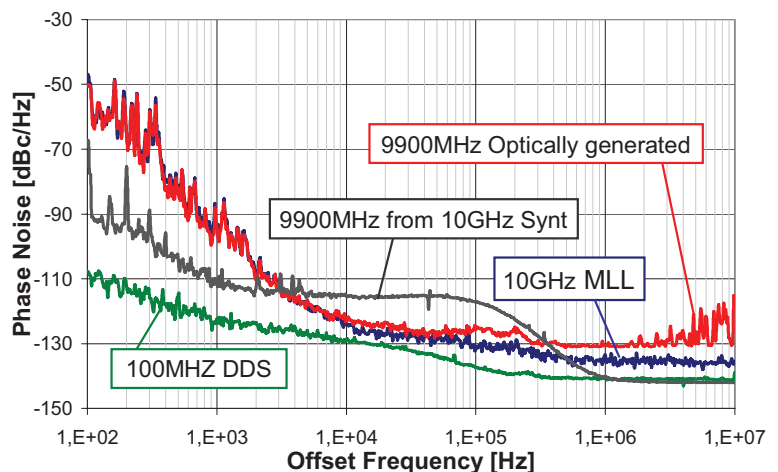

(a)

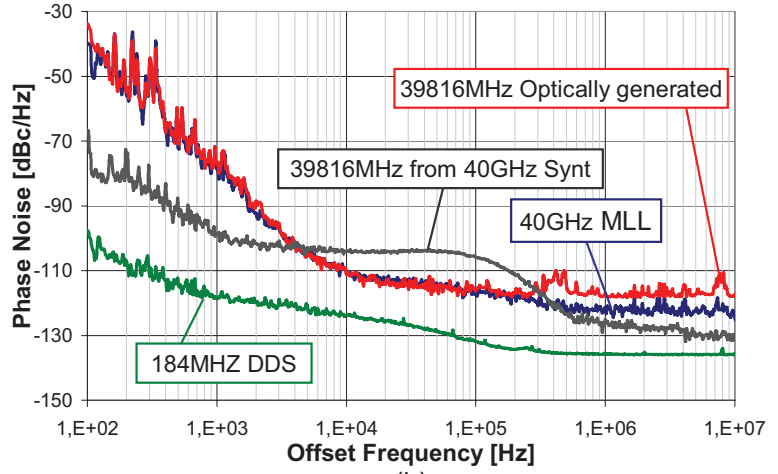

(b)

Fig. 4. Phase noise of the generated carriers, compared with the non-shifted carrier from the MLL, with the shifting signal from the DDS, and with the carrier obtained by shifting the signal from a synthesizer in a mixer. (a) Carrier at $9.9 \mathrm{GHz}$. (b) Carrier at $39.816 \mathrm{GHz}$.

in an optical phase-lock loop [14]. Moreover, a MLL with improved noise performance could be used, as for example the semiconductor MLL reported in [15], allowing a reduction of the phase noise.

Nevertheless, integrating the phase noise curves from $1 \mathrm{kHz}$ to $1 \mathrm{MHz}$, the measured rms time jitter is $14.2 \mathrm{fs}$ for the optically generated carrier and $15.3 \mathrm{fs}$ for the conventionally generated one, confirming the noise performance of the proposed scheme.

By changing the RF-filter and resetting the DDS output to $184 \mathrm{MHz}$, a $39.816 \mathrm{GHz}$ carrier is produced. Fig. 4(bottom) shows that the phase stability of the carrier at $39.816 \mathrm{GHz}$ is also not affected by the shifting process, and the comparison with the synthesizer curve confirms that the proposed scheme is suitable for generating high-frequency signals for coherent radar systems, performing better than an electronic-based approach. In this case the integration of the curves gives an rms time jitter of $16.1 \mathrm{fs}$ for the optically generated carrier and $16.3 \mathrm{fs}$ for the conventionally generated one.

In order to demonstrate the effectiveness of the proposed scheme, it has been characterized by generating radar pulses implementing the most used pulse-compression techniques. The DDS is first set to generate a $25 \mathrm{MHz}$ linear chirp around $100 \mathrm{MHz}$, i.e. a linear frequency sweep from $87.5 \mathrm{MHz}$ to $112.5 \mathrm{MHz}$, and the RF filter centered at $9.9 \mathrm{GHz}$ is used. The choice of such a frequency deviation is due to the limited bandwidth of the available RF filters and of the DDS, but in principle the only bandwidth limitation is given by the

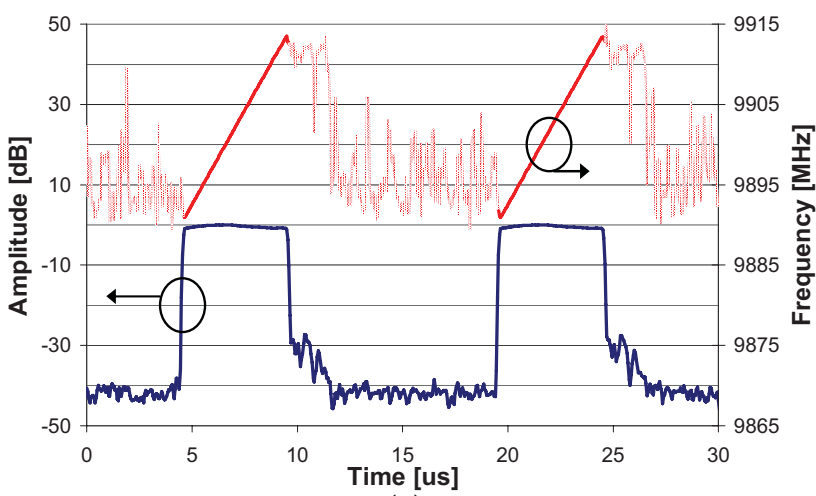

(a)

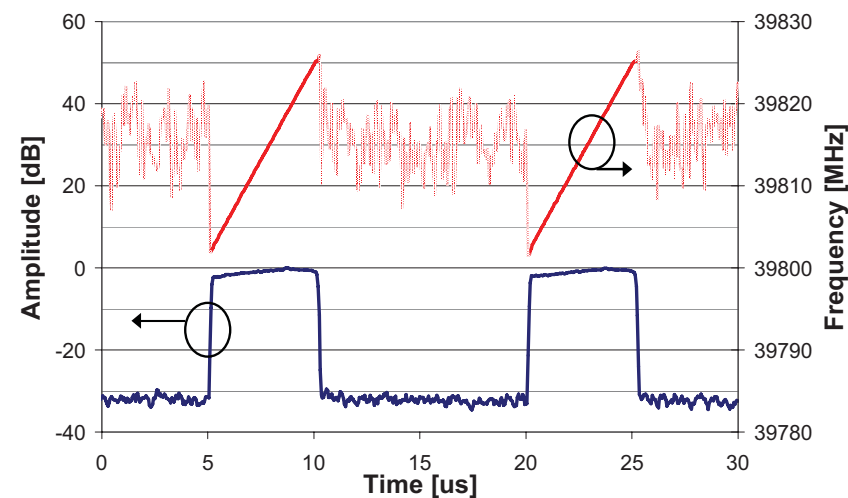

(b)

Fig. 5. Frequency and amplitude transients of the signals measured with the SSA. (a) Carrier at $9.9 \mathrm{GHz}$. (b) Carrier at $39.816 \mathrm{GHz}$.

precise DDS (hundreds of $\mathrm{MHz}$ ) and by the MLL repetition rate, which must be high enough to prevent overlaps between different carrier frequencies.

The modulated signal obtained with the proposed setup is analyzed by the SSA in terms of frequency and amplitude transients, as reported in Fig. 5(top). The graph shows that the signal generated by the DDS is perfectly upconverted to $9.9 \mathrm{GHz}$, producing a linearly chirped pulse across $25 \mathrm{MHz}$. This proves that the proposed schemes is capable of effectively upconvert amplitude- and phase-modulated signals. Moreover, the pulsed amplitude shows an extinction ratio of about $40 \mathrm{~dB}$, even if a minor ripple is present on the right side of the pulses, caused by the narrow bandwidth of the RF filter. Finally, Fig. 6(top) reports the electrical spectrum, acquired with a $40 \mathrm{GHz}$-bandwidth electrical spectrum analyzer (ESA), of the generated signal, compared with the simulated spectrum of an equivalent ideal chirped signal, showing an almost perfect match. The spectral broadening of the compressed pulse with respect to the unchirped RF pulse of the same time duration is reported in Fig. 6(top). Since the spatial resolution $\Delta \mathrm{R}$ of a radar system is proportional to the inverse of the signal bandwidth $\mathrm{B}(\Delta R=c / 2 B)$, if we use a chirped signal with a frequency deviation $\Delta \mathrm{F}$ the range resolution becomes $\Delta R=c /(2 \Delta F)$. In our case, where the signal bandwidth of the uncompressed pulse is $200 \mathrm{kHz}$ (a $5 \mu$ s pulsed signal) and the chirp frequency deviation is $25 \mathrm{MHz}$, we obtain an increased radar resolution from $750 \mathrm{~m}$ to $6 \mathrm{~m}$.

By setting the DDS to generate a chirped signal centered at $184 \mathrm{MHz}$ and replacing the RF filter, a modulated pulsed 
signal at $39.816 \mathrm{GHz}$ is produced. The analyses reported above are applied for this case as well. In Fig. 5(bottom), a lower extinction ratio is reported on the amplitude transients with respect to the case at $9.9 \mathrm{GHz}$, which is due to a higher noise floor of the SSA when measuring high-frequency signals. The electrical spectrum reported in Fig. 6(bottom) appears to be tilted with respect to the ideal case, with the spectral components at lower frequencies slightly weaker than the higher frequencies. This is caused by the analog bandwidth of the DDS which is limited to 200MHz: since the chirped signal runs from $196.5 \mathrm{MHz}$ to $171.5 \mathrm{MHz}$, its high-frequency components are slightly attenuated and transferred to the carrier at $39.816 \mathrm{GHz}$.

The effectiveness of the employed pulse-compression technique is also proven by the autocorrelation function (AF), calculated from a single shot trace acquired with a 40Gsample/s real-time oscilloscope, and reported in Fig. 7. From the graph the obtained compression is clearly evident, especially compared with the AF envelope of an unchirped pulse (dotted line). Due to the limited bandwidth of the oscilloscope the AF is related to the $9.9 \mathrm{GHz}$ case only but, as demonstrated by the SSA and ESA traces, there are no reasons to suppose that the $39.816 \mathrm{GHz}$ case would be different.

Another frequently exploited pulse-compression technique is the binary phase coding using the Barker codes. These are particular sequences of $\mathrm{N}$ values $\mathrm{a}_{j}$ in the set $(-1,+1)$ for $j=1,2, \ldots, N$, such that

$$
\left|\sum_{j=1}^{N-m} a_{j} a_{j+m} \leq 1\right|
$$

for $1 \leq \mathrm{m}<\mathrm{N}$. They are applied to radar pulses by dividing the pulse in $\mathrm{N}$ subpulses of equal duration, and modulating the carrier phase in each subpulse by $0^{\circ}$ or $180^{\circ}$ according to the adopted Barker code. Thanks to the coding, these kind of radar pulses show an autocorrelation peak $\mathrm{N}$ times narrower, and autocorrelation sidelobes $\mathrm{N}$ times smaller [1], than the $\mathrm{AF}$ of the uncompressed pulse. This means that, by analysing the cross-correlation between the detected radar echo and the transmitted pulse, an N-times increased resolution can be obtained with respect to the unmodulated radar pulse, with a sensitivity improvement of $\log _{10} \mathrm{~N} \mathrm{~dB}$, without reducing the pulse duration and thus avoiding an increase in the transmitted peak power. The code length $\mathrm{N}$ can range from 2 to 13 bits and determines the range resolution improvement according to the equation $\Delta \mathrm{R}=\mathrm{c} / 2 \mathrm{BN}$. In our experiment we have used the longest code, i.e. $+1,+1,+1,+1,+1,-1,-1,+1,+1$, $-1,+1,-1,+1$, since it provides the best compression factor of $1 / 13$ with respect to the uncompressed pulses.

The DDS is set to generate a coded pulse at $100 \mathrm{MHz}$, and the $9.9 \mathrm{GHz} \mathrm{RF}$ filter is used. The phase-modulated signal obtained is analyzed by the SSA in terms of phase and amplitude transients, as reported in Fig. 8(top).

The graph shows that the signal generated by the DDS is again perfectly upconverted to $9.9 \mathrm{GHz}$, producing $180^{\circ}$ phase jumps in the pulses. Fig. 9(top) reports the electrical spectrum of the generated signal, compared with the simulated spectrum of an equivalent ideal coded signal, showing again an almost

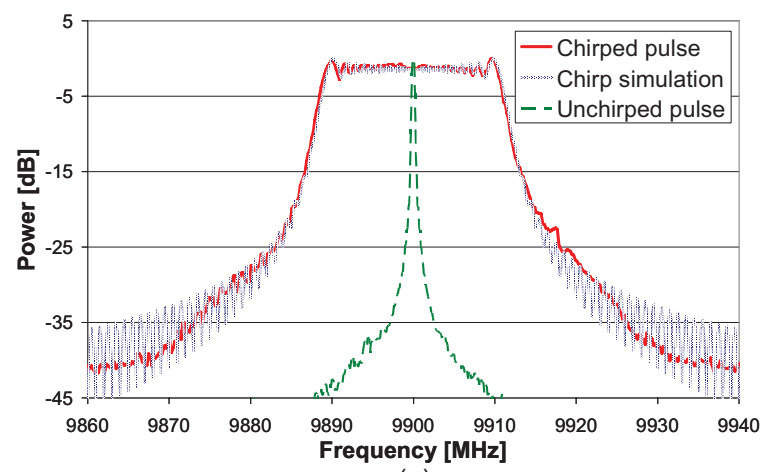

(a)

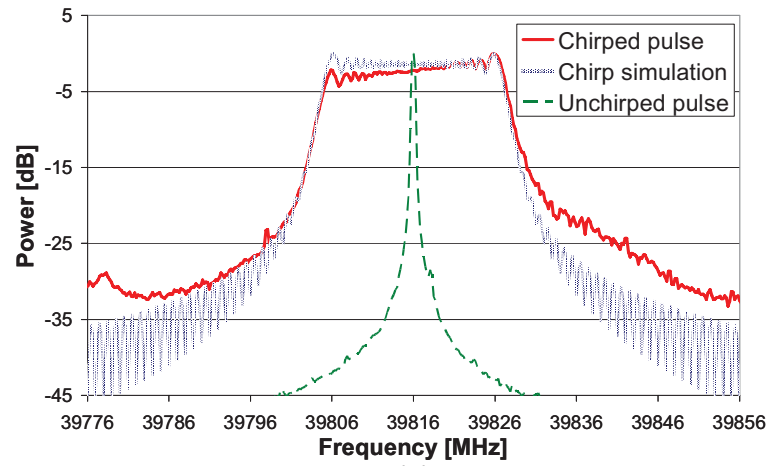

(b)

Fig. 6. Measured electrical spectrum (RBW $100 \mathrm{kHz}$ ) of the chirped pulses, compared with the unchirped RF pulses and the simulation of an ideal chirp. (a) Carrier at $9.9 \mathrm{GHz}$. (b) Carrier at $39.816 \mathrm{GHz}$.

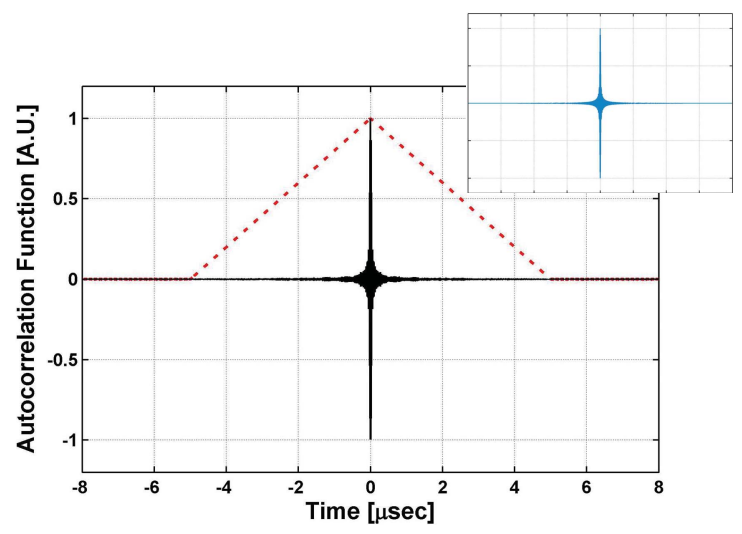

Fig. 7. AF of the chirped pulses extracted from the real-time oscilloscope trace, compared with the AF envelope (dotted line) of an unmodulated pulse. Inset: the simulated $\mathrm{AF}$ of the ideal chirped pulse.

perfect match. The spectral broadening of the compressed pulse with respect to the uncoded RF pulse of the same time duration, reported in Fig. 9(top), reveals an increased radar resolution up to $57.7 \mathrm{~m}$.

By setting the DDS to generate a phase-coded signal at $184 \mathrm{MHz}$ and replacing the RF filter, a phase-coded pulsed signal at $39.816 \mathrm{GHz}$ is produced. The analyses reported above are applied for this case as well, and are shown in Fig. 8(bottom) and Fig. 9(bottom).

In Fig. 10 the autocorrelation function of the generated Barker coded pulse at $9.9 \mathrm{GHz}$ is shown. As expected from the radar theory, the normalized amplitude presents sidelobe peaks at $0.07(1 / \mathrm{N})$ and corresponds well to the simulated 


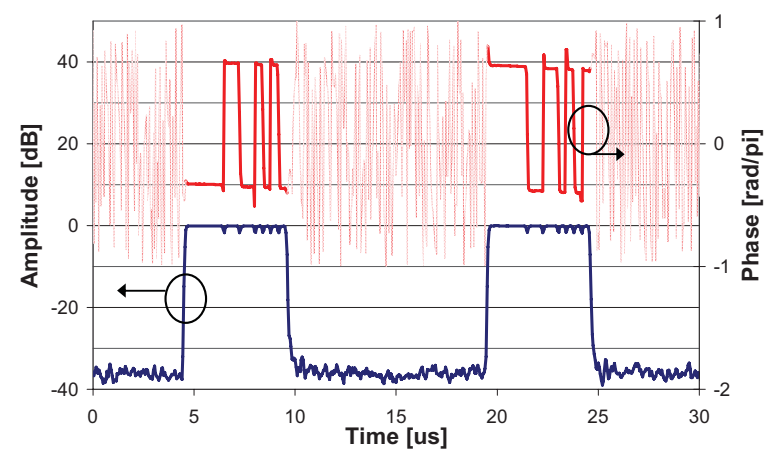

(a)

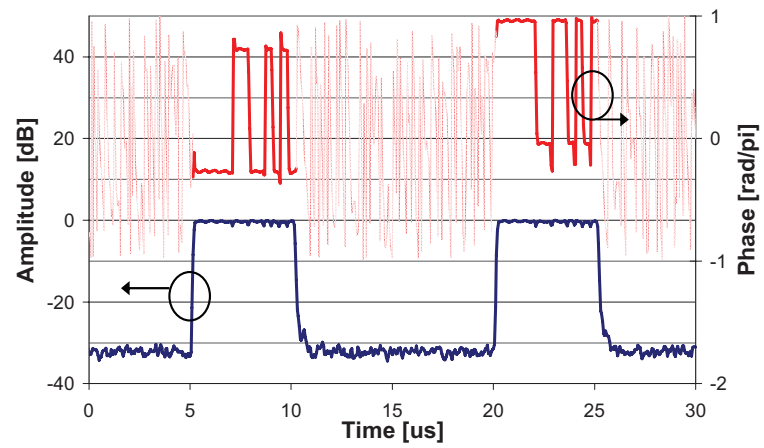

(b)

Fig. 8. Phase and amplitude transients of the signals measured with the SSA. (a) Carrier at $9.9 \mathrm{GHz}$. (b) Carrier at $39.816 \mathrm{GHz}$.

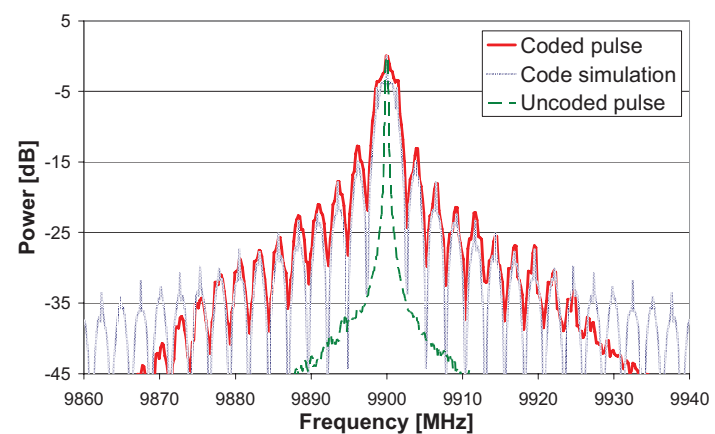

(a)

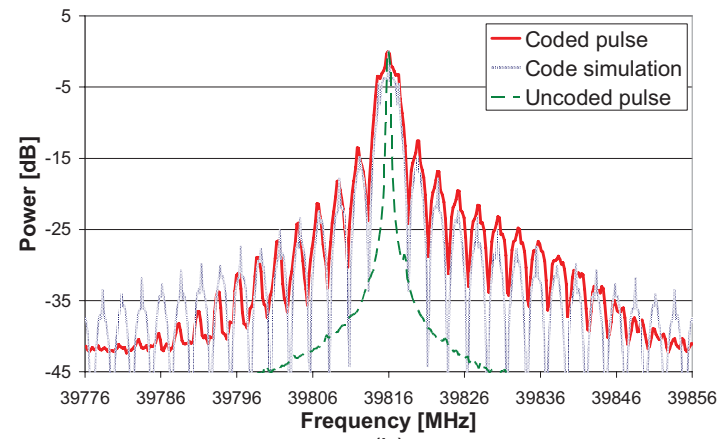

(b)

Fig. 9. Measured electrical spectrum (RBW $100 \mathrm{kHz}$ ) of the phase-coded pulses, compared with the uncoded RF pulses and the simulation of an ideal 13-bit Barker code. (a) Carrier at $9.9 \mathrm{GHz}$. (b) Carrier at $39.816 \mathrm{GHz}$.

AF of the sequence shown in the inset. So the suitability of the proposed scheme for producing phase-coded radar signals is confirmed. With the particular code exploited, the range resolution is improved by a factor of 13 from $750 \mathrm{~m}$ (relative to the unmodulated pulses) to $57.7 \mathrm{~m}$.

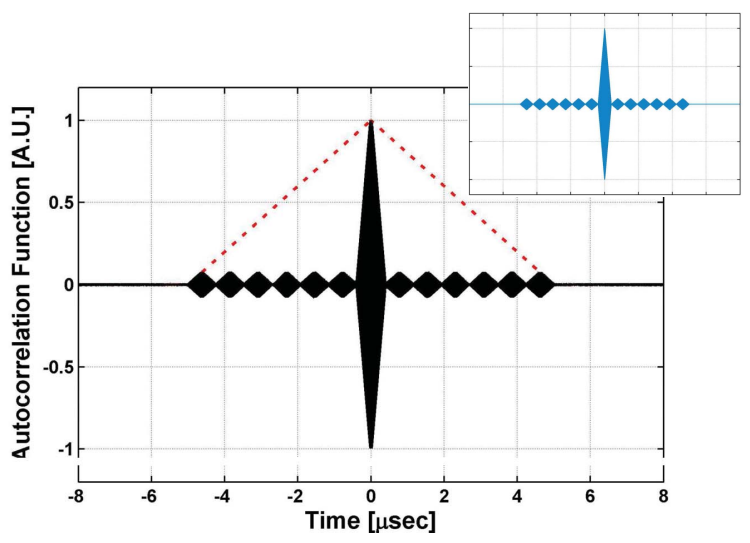

Fig. 10. Autocorrelation function of the Barker-coded pulses extracted from the real-time oscilloscope trace, compared with the envelope (dotted line) of an unmodulated pulse. Inset: the simulated AF of the ideal coded pulse.

\section{CONCLUSion}

The proposed method for the generation of frequency-agile phase modulated RF signals can be of help in a large number of applications. Besides coherent radars, it can be used wherever phase modulated RF signals are needed, as for example in radio-over-fiber systems for the generation of complex modulation formats (e.g., 64QAM), or in the removal of the range ambiguity in radars and lidars [16].

Different applications and scenarios may require different carrier frequencies and modulation bandwidths, so it is necessary to choose the MLL's repetition rate carefully. A MLL with high repetition rate can allow wide-band modulation, since carrier's overlap is avoided, but it would reduce the number of available frequencies since the bandwidth of the commercial photodiodes is limited to about $100 \mathrm{GHz}$. On the other hand, a low repetition rate MLL can allow a dense set of available frequencies, but reduces also the possible modulation bandwidth.

As demonstrated by our measurements, the phase stability of the generated RF pulses depends on the timing jitter of the original MLL. Therefore, the better the MLL, the better the RF signal. As it is evident, if an ultra-low-noise MLL is used as laser source [15], then the noise-limiting element becomes the exploited DDS.

Although the proposed system requires readily available components, photonic hybrid integration my be helpful to further reduce the complexity of the setup [17]. This way all the optical path could be replaced by a single monolithic structure with reduced footprint, allowing its implementation even in mobile systems.

In conclusion, we have proposed an innovative scheme that exploits photonics for generating arbitrary phase-modulated RF pulses with flexible carrier frequency, and phase stability suitable for coherent radar systems. The use of a lowfrequency high-quality DDS permits the maximum flexibility in the generation of software-defined modulating signals which are transferred to the modes of a MLL, without affecting their reciprocal phase stability. The digital signals are generated at an intermediate frequency, so that after detecting the optical 
signal, phase-modulated RF pulses can be obtained close to any multiple frequency of the MLL repetition rate, even at frequency in the EHF band. The reported experimental results confirm the generation of linearly chirped and phase-coded RF pulses with carrier frequencies of about $10 \mathrm{GHz}$ and $40 \mathrm{GHz}$ with very high phase stability, and demonstrate a spectral broadening suitable for pulse compression techniques. The flexibility of the scheme allows to implement any kind of modulation (e.g., Frank codes) at any carrier frequency. By properly choosing the MLL repetition rate, frequency agility can be implemented on close or far frequencies (as in frequency hopping or in multifunctional radars, respectively), or even both, with the advantage of a single MLL instead of a series of electronic oscillators for each carrier frequency. The carriers can be generated simultaneously or alternately, or even changed continuously. The modulating signal can also be changed meanwhile, implementing a waveform diversity technique.

Therefore the proposed technique allows the practical realization of compressed pulses for coherent radars over a wide carrier frequency range, allowing the development of softwaredefined systems with improved functionalities.

\section{REFERENCES}

[1] M. L. Skolnik, Introduction to Radar Systems. New York: McGraw-Hill, 1980.

[2] V. Ravenni, "Performance evaluations of frequency diversity radar system," in Proc. Eur. Microw. Conf., Oct. 2007, pp. 436-439.

[3] J. J. Zhang and A. Papandreou-Suppappola, "MIMO radar with frequency diversity," in Proc. Waveform Div. Desgin Conf., 2009, pp. 208212.

[4] J. Sun, Y. Dai, X. Chen, Y. Zhang, and S. Xie, "Stable dual-wavelength DFB fiber laser with separate resonant cavities and its application in tunable microwave generation," IEEE Photon. Technol. Lett., vol. 18, no. 24 , pp. 2587-2589, Dec. $15,2006$.

[5] L. Goldberg, R. D. Esman, and K. J. Williams, "Generation and control of microwave signals by optical techniques," IEE Proc. J. Optoelectron., vol. 139, no. 4, pp. 288-295, Aug. 1992.

[6] G. Serafino, P. Ghelfi, G. E. Villanueva, J. Palaci, P. Perez-Millan, J. L. Cruz, and A. Bogoni, "Phase and amplitude stability of EHF-band radar carriers generated from an active mode-locked laser," IEEE J. Lightw. Technol., vol. 29, no. 23, pp. 3551-3559, Dec. 1, 2011.

[7] J. D. McKinney, D. E. Leaird, and A. M. Weiner, "Millimeter-wave arbitrary waveform generation with a direct space-to-time pulse shaper," Opt. Lett., vol. 27, no. 5, pp. 1345-1347, Aug. 2002.

[8] J. Chou, Y. Han, and B. Jalali, "Adaptive RF-photonic arbitrary waveform generator," IEEE Photon. Technol. Lett., vol. 15, no. 4, pp. 581583, Apr. 2003.

[9] I. S. Lin, J. D. McKinney, and A. M. Weiner, "Photonic synthesis of broadband microwave arbitrary waveform applicable to ultrawideband communication," IEEE Microw. Wireless Compon. Lett., vol. 15, no. 4, pp. 226-228, Apr. 2005.

[10] H. Chi and J. P. Yao, "An approach to photonic generation of high frequency phase-coded RF pulses," IEEE Photon. Technol. Lett., vol. 19, no. 10, pp. 768-770, May 15, 2007.

[11] Z. Li, W. Li, H. Chi, X. Zhang, and J. Yao, "Photonic generation of phase-coded microwave signal with large frequency tunability," IEEE Photon. Technol. Lett., vol. 23, no. 11, pp. 712-714, Jun. 1, 2011.

[12] P. Ghelfi, F. Scotti, F. Laghezza, and A. Bogoni, "Photonic generation of phase-modulated RF signals for pulse compression techniques in coherent radars," IEEE J. Lightw. Technol., vol. 30, no. 11, pp. 16381644, Jun. 1, 2012

[13] T. Yilmaz, C. M. DePriest, T. Turpin, J. H. Abeles, and P. J. Delfyett, "Toward a photonic arbitrary waveform generator using a modelocked external cavity semiconductor laser," IEEE Photon. Technol. Lett., vol. 14, no. 11, pp. 1608-1610, Nov. 2002.

[14] L. Banchi, F. Rossi, M. Ferianis, A. Bogoni, L. Potì, and P. Ghelfi, "Synchronization of $3 \mathrm{GHz}$ repetition rate harmonically mode-locked fiber laser for optical timing applications," in Proc. DIPAC, 2007, pp. $1-3$.
[15] P. J. Delfyett, S. Gee, F. Quinlan, P. Juodawlkis, and J. Plant, "Low noise and stabilized mode-locked diode lasers for arbitrary waveform generation," in Proc. IEEE Lasers Electro-Opt. Soc. Annu. Meet., Nov. 2008, pp. 352-353.

[16] M. U. Piracha, D. Nguyen, D. Mandridis, T. Yilmaz, I. Ozdur, S. Ozharar, and P. J. Delfyett, "Range resolved lidar for long distance ranging with sub-millimeter resolution," Opt. Express, vol. 18, no. 7, pp. 7184-7189, Mar. 2010.

[17] S. Cheung, J. Baek, R. P. Scott, N. K. Fontaine, F. M. Soares, X. Zhou, D. M. Baney, and S. J. B. Yoo, "1-GHz monolithically integrated hybrid mode-locked InP laser," IEEE Photon. Technol. Lett., vol. 22, no. 24 , pp. 1793-1795, Dec. 15,2010

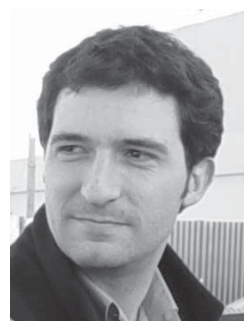

Paolo Ghelfi was born in Mantova, Italy, in 1975. He received the M.S. degree in electronics engineering from the University of Parma, Parma, Italy, in 2000.

He was a Grantee with the Optical Communications Laboratory, Parma University, Parma, from 2000 to 2001. Since 2002, he has been with the National Photonic Networks Laboratory, National Interuniversity Consortium for Telecommunications, Pisa, Italy. His current research interests include fiber optical transmissions and all-optical processing.

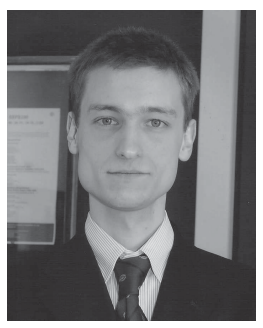

Filippo Scotti was born in Milano, Italy, in 1985. He received the Bachelors and the M.S. degrees in physics engineering from the Politecnico of Milano, Milano, in 2007 and 2009, respectively.

$\mathrm{He}$ was with PGT-Photonics, Milano, in 2009 , for a 6-month internship. Since 2010, he has been with the National Photonic Networks Laboratory, National Interuniversity Consortium for Telecommunications, Pisa, Italy. His current research interests include fiber optic transmissions, all-optical signal processing, and ultrashort optical pulse sources and

their RF application.

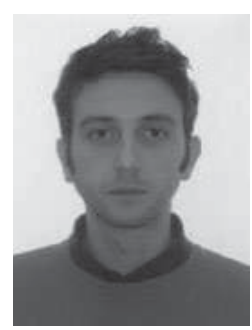

Francesco Laghezza was born in Taranto, Italy, in 1982. He received the M.S. degree in telecommunication engineering from the University of Pisa, Pisa, Italy, in April 2009, where he is currently pursuing the Ph.D. degree in remote sensing.

He was a Postgraduate Research Assistant with the National Interuniversity Consortium for Telecommunications, Pisa, from 2009 to 2010. His current research interests include full digital electro-optical radar design, space debris radar detection, and array processing.

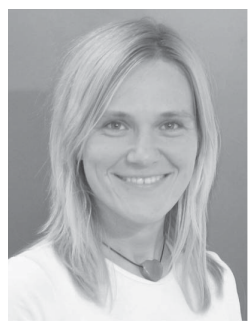

Antonella Bogoni received the Ph.D. degree from the University of Parma, Parma, Italy, in 2004.

She is currently the Head of Research with National Interuniversity Consortium for Telecommunications, Integrated Research Center for Photonic Networks and Technologies, Pisa, Italy. She has been a scientific coordinator for national and international projects and has been involved in several EU and national projects. She has authored or co-authored more than 200 papers in international journals and conferences and holds more than 40 international patents. She has participated in revision committees of international conferences. She is a reviewer for international journals and for the European Commission within FP7. Her current research interests include ultrafast alloptical signal processing and digital photonics.

Dr. Bogoni was the recipient of the Fulbright Advanced Research Scholarship for the Project Design and Implementation of a $640 \mathrm{~Gb} / \mathrm{s}$ OTDM System from the University of Southern California, CA, in 2009. 